\title{
Observation of the temperature field dynamics by the thermospectroscopic imaging
}

\author{
by M. Ryu*, J. Morikawa* \\ *Tokyo Institute of Technology, Department of Material Science and Engineering, Meguro-ku Ookayama 2-12-1, \\ 152-0033, Tokyo, Japan, Morikawa.j.aa@m.titech.ac.jp
}

\begin{abstract}
Non-contact qualitative measurement of the temperature field with Infrared camera based on the thermal radiation measurement has been extensively investigated for the material thermal characterization and observation of the thermal energy transfer in a microscale. Non-contact temperature measurement has the advantage that the sensor itself does not disturb the temperature field in the sample. However, the estimation of the absolute value of the temperature from the thermal radiation is not always possible, especially in the concentration distributed field, such as in a liquid mixture or in the co-existence region of the several phases of the material. This is due to the emissivity distribution of the sample.

Microscale thermospectroscopic imaging has been developed for the simultaneous imaging of the infrared spectrum and thermal emission in mid IR region with microscale special resolution. This imaging method was further extended for the quantitative analysis of the concentration and temperature field in organic molecular system, such as phase transition or the chemical reaction in the microfluidics. This imaging method for the temperature estimation is based on the spatial correlation between emissivity distribution and concentration distribution. In this study, generalized analysis method of the thermospectroscopic imaging was investigated, and the analysis of the phase transition and microscale mixing of the liquids are explained by a unified equation for the concentration and temperature dependent IR camera signal.
\end{abstract}

\section{Introduction}

The thermal imaging technique using infrared (IR) camera relies on the Stefan-Boltzmann law, where the signal depends not only on the temperature but also on the emissivity of the emitting materials. For the determination of the absolute temperature, the calibration process of the emissivity is needed for each material before the thermal imaging. This process becomes quite complex when the sample has the in-plane distribution of the emissivity. In this case, thermal emission signal is the superimposed image of the distributions of the temperature and the emissivity. This multi-variable function-like behavior of thermal emission signal limits the absolute temperature estimation in complex sample with thermal imaging.

In the case of the exothermic phenomena in a mixture, such as in the mixed solutions or in the microfluidics, the thermal emission signal detected by the IR camera is dominated by the microscale concentration distribution. In order to extract the temperature and concentration image separately from the observed signals, the combined imaging system of the thermal emission and the IR transmission spectroscopy (thermo-spectroscopy) was proposed and applied the organic material phase transition and the liquid mixture in the microfluidics $[1,2]$.

Two reported examples are different exothermic phenomena in a microscale however, these systems can be analyzed by the same image calculation procedure. In this study, the generic formulation of the thermo-spectroscopy is presented and examined according to the result of the phase transition and the liquid mixture process. Furthermore, the method is tested not only for the two components system but also for the three components system at the co-flow interface between reactive liquids in the microfluidics.

\section{Experiment}

The device consists of a diffraction grating monochromator and a reflection condenser lens of 3 times magnification originally designed with JASCO RF-10 and IR camera (Indigo, Phoenix, InSb 320 x 256 pixels) with an originally designed optics for a microscale imaging. The wavelength resolution of the spectrum is $10 \mathrm{~nm}$, confirmed with the narrow bandpass filters (Spectrogon, NB3550).

For the simultaneous IR spectroscopic and thermal imaging, the pulse signal whose frequency is exactly twice as large as chopper frequency is required for the shutter control of IR camera. The shutter control signal was generated by an arbitrary waveform generator (Pragmatic Instruments Inc., 2711A), which was synchronized with a chopper and generated the sequence, including two pulses. For the simultaneous imaging of $128 \times 128$ pixels mode, the chopper was set at $100 \mathrm{~Hz}$ frequency and the frame rate of the IR camera was set at $200 \mathrm{~Hz}$.

\section{Method}

Generally, the thermal emission $E$ of the mixture is a function of the temperature and the concentration, since the emissivity is depending on the concentration of each component of the mixture. Therefore, the total differential of the thermal emission signal can be written as follows, 


$$
\mathrm{d} E=\left(\frac{\partial E}{\partial T}\right)_{C} \mathrm{~d} T+\left(\frac{\partial E}{\partial C}\right)_{T} \mathrm{~d} C
$$

where $T$ is the temperature $(\mathrm{K})$, and $C$ is a concentration $(\mathrm{mol} / \mathrm{l})$. For the practical measurement, each differential in Eq. (1) can be described by the finite difference between measurement points and the reference point.

To estimate the absolute temperature, the thermal emission signal at each temperature and at each concentration are measured, prior to the experiment as a calibration procedure. From the calibration results, the calibration surface in the $E$, $T, C$ space can be obtained, and $\left(T_{0}, C\right)$, which is the thermal emission signal intensity without the exothermic heat, can be calculated. The difference between calculated value $E\left(T_{0}, C\right)$ and measurement value $E(T, C)$ is a function of $\Delta T$, and can be described as follows,

$$
E(T, C)-E\left(T_{0}, C\right)=\left\{\left(\frac{\partial E}{\partial T}\right)_{C}+\frac{\partial}{\partial T}\left(\frac{\partial E}{\partial C}\right) \Delta C\right\} \Delta T
$$

where $C_{0}$ is the pure concentration of the sample (mol/l), $\Delta T=T-T_{0}$, and $\Delta C=C-C_{0}$. Since two partial differential coefficients in Eq. (2) can be obtained from the results of calibration procedure, $\Delta T$ value at each pixel in the image can be estimated.

In this study, the co-flow interface between water and ethanol, water and $\mathrm{HCl}$ solution, and styrene radical polymerization were chosen for the example of two components system, and the neutralization reaction of acid and base was chosen for the three components concentration distributed system of $\mathrm{HCl}, \mathrm{NaOH}$, and $\mathrm{NaCl}$ solution. The conventional micro-scale thermos-spectroscopic imaging set up for microfluidics chip [3] was used for the experiment and $E$ image were obtained from the thermal emission image of the set up and $C$ image were obtained from spectroscopic image of the system. For the phase transition, solid-rotator-liquid transition of $n$ - tetracosane $\left(\mathrm{C}_{24} \mathrm{H}_{50}, \mathrm{C} 24\right)$ was chosen for the investigation.

\section{Results and discussion}

In figure 1, the result of the absolute temperature estimation for the liquid mixture and the phase transition were shown. The results were compared with the dissolution heat or the latent heat in the literatures and the validity of the analysis process was discussed.
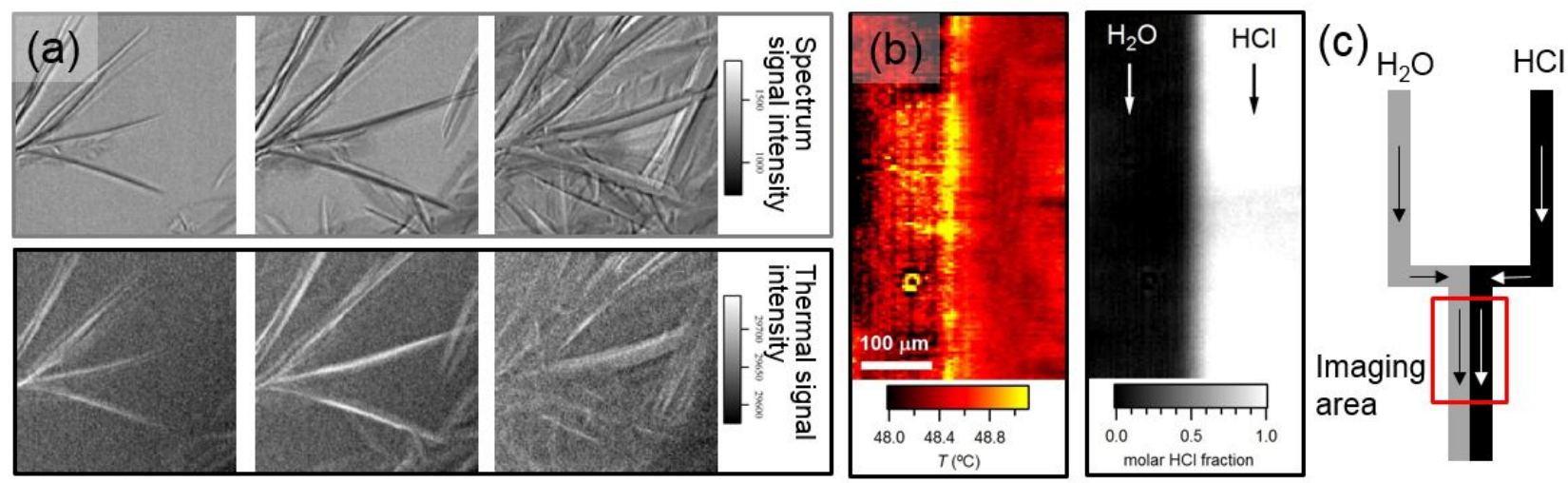

Fig. 1: Temperature estimation of the concentration distributed exothermic system. (a) Thermal images and IR spectroscopic images during phase transition from liquid to rotator phase of C24. (b) Molar $\mathrm{HCl}$ fraction field image calculated from $3800 \mathrm{~nm} I R$ spectroscopic image and the temperature image at the co-flow interface between water and $\mathrm{HCl}(2 \mathrm{~mol} / \mathrm{L})$. (c) Schematic description of the imaging area in the microfluidics.

\section{REFERENCES}

[1] M. Romano, M. Ryu, J. Morikawa, J.C. Batsale, C. Pradere, "Simultaneous microscopic measurements of thermal and spectroscopic fields of a phase change material", Infrared Physics \& Technology, 76, 65-71, (2015)

[2] M. Ryu, M. Romano, J.C. Batsale, C. Pradere, J. Morikawa, "Microscale spectroscopic thermal imaging of $n-$ alkanes", Quantitative InfraRed Thermography J., 10.21611 (2016)

[3] M. Ryu, J.A. Kimber, T. Sato, R. Nakatani, T. Hayakawa, M. Romano, C. Pradere, A.A. Hovhannisyan, S.G. Kazarian, J. Morikawa, "Infrared thermo-spectroscopic imaging of styrene radical polymerization in microfluidics", Chem. Eng. J., accepted, (2017) 